\title{
VUE DE FRANCE
}

\section{L'OBUVRE DIALOGIQUE DE FRANÇOIS CANTAGREL}

\begin{abstract}
Nous remercions l'auteur de cet essai, ainsi que la Directrice de la revue philosophique Corpus, et les éditions Fayard, editeurs de la Collection d'oeuvres de Philosophie, Corpus, qui nous ont donné la permission d'utiliser ce texte.
\end{abstract}

François (Jean, Félix) Cantagrel est né à Amboise le 27 juin 1810. D'origine bourgeoise, il vint, en 1827, continuer ses études à Paris. Esprit éclectique, il fait ses débuts littéraires dans l'Artiste, auquel il collabore de 1834 à 1838, tout en poursuivant des études de droit et d'architecture. En 1838, il est ingenieur civil, conducteur des Ponts et Chaussées, lorsque la lecture des ouvrages de Charles Fourier et de Victor Considérant l'amène à se consacrer exclusivement à la propagation de la doctrine sociétaire.

Son premier et principal ouvrage, publie pour la première fois en 1841, fut Le Fou du Palais-Royal. Procédant "en seize Propos et trente-deux Sous-Propos (Série à trente-deux touches), comme défilent en parade les seize Tribus et trente-deux Choeurs de la Phalange d'attraction", l'auteur y développe les idées de Fourier sous forme de dialogue, et s'efforce de répondre aux objections qu'elles soulevvent.

Ami de Considérant, collaborateur puis gerant du journal phalanstérien La phalange, qui devint en 1843 la Démocratie Pacifique, il fut poursuivi en cette qualite, au mois d'aout 1847, comme responsable ou auteur d'articles "outrageant la morale publique." De 1843 à 1848, il publia diverses brochures: Les enfants au Phalanstère, une étude sur les colonies agricoles, Mettray et Ostwald, une autre sous le titre: Quinze millions à gagner sur les bords de la Cisse, une étude remarquée sur l'Organisation des travaux publics et la Reforme des Ponts et Chaussées (1847).

Fervent républicain, il reçut à la Démocratie Pacifique, le 3 février 1848, les étudiants de Paris qui allaient porter aux journaux leurs pétitions pour le rétablissement des chaires de MM. Michelet et Quinet et leur adressa ces paroles: "nous sommes vos aînés de $1830 . .$. nous avons 
fait notre devoir alors; à vous de faire le vôtre aujourd'hui. Amis, il faut jeter un trait d'union entre 1830 et 1848."

En mai 1849, les électeurs du Loir-et-Cher l'envoyèrent à l'Assemblée législative. Il n'y siegera que six semaines: traduit devant la Haute Cour de Versailles pour sa participation à l'insurrection du 13 juin 1849 , il trouvera un refuge en Belgique. Condamné par contumace à la déportation à perpétuité, il passa en Angleterre, visita les Etats-Unis, puis revint en 1851 en Belgique, où il publia trois ouvrages sur la question religieuse: Comment les dogmes commencent (1857); Nécessité d'un nouveau symbole (1859); D'où nous venons, où nous allons, où nous sommes (1858). A Neufchâtel, où il dirige en 1859 le journal L'Indépendant, il publia encore l'Election véridique ou la Sincérité représentative assurée par le vote secret et libre.

Revenu en France après l'amnistie de 1859, il fut candidat en 1863 et en 1869, où il tenta sa chance, sans empêcher l'election de Jules Favre, dans la septieme circonscription de la Seine. Sa profession de foi se voulait conciliatrice, il était "le seul dont le nom puisse grouper à la fois les démocrates radicaux et les socialistes." La même année, il défendit dans La Réforme les idées coopératistes. Cette volonté de conciliation se manifesta à nouveau au moment de la Commune. Proche de V. Schoelcher et de V. Considerant, qui multiplient en avril 1871 leurs tentatives pour arrêter les hostilités, il ne joue cependant aucun rôle dans les évènements, même si Félix Piat propose sa candidature dans le IXe arrondissement aux élections complémentaires du 16 avril 1871. En fait, éloigné de la capitale, il est alors à Nantes où il a repris le journal L'Union démocratique, fondée en 1865 par le docteur Guépin. Il y patronne Georges Clémenceau qu'il introduit dans des clubs ouvriers. Devenu radical, franc-maçon, bientôt vénérable de la loge Les Amis Inséparables, la figure ornée d'une barbe blanche, patriarcal d'aspect, il se fait élire, le 30 juillet 1871, conseiller municipal de Paris, sur un programme démocratique radical. Cette élection écourta son séjour à Sainte-Pélagie, où il fut pourtant incarcéré le 25 septembre en application du jugement rendu le 9 juin 1871 aux assises de Nantes, par lequel il était condamné à six mois de prison pour "excitation à la haine et au mépris du gouvernement et désobéissance aux lois." Libéré sur les instances du prefet Léon Say, il put siéger et devint vice-président du Conseil Général de la Seine en 1872, puis député du XIIIe arrondis-sement en 1876. Il remercia ses électeurs en leur assurant qu'il defendrait à la Chambre "lorganisation régulière et progressive de la République démocratique et 
sociale." Député radical, votant pour l'amnistie, contre le Concordat et les expéditions coloniales, il s'y employa jusqu’à sa mort, en 1887.

S'il ne s'est jamais limite à un role purement doctrinal, François Cantagrel doit avant tout sa notoriété au succès de son oeuvre maîtresse, Le Fou du Palais-Royal, que les fouriéristes rééditèrent en 1845. Fameuse de son vivant, l'oeuvre étonne toujours par son humour et sa liberté d'expression. D'un simple "Manuel ou Memento Phalanstérien" (Septième Propos), il a su faire, en évitant "la forme didactique simple" (Deuxième Propos), une magistrale et originale introduction à la lecture de Fourier.

Charles Pellarin relate ${ }^{1}$ - que Fourier, pendant les dernières années de sa vie, avait coutume de passer "une ou deux heures chaque jour dans le cabinet de lecture de la Rotonde au Palais-Royal, pour se tenir au courant des évènements du jour et des sujets de discussion soulevés dans la presse." C'est ce lieu que Cantagrel a retenu pour situer les propos familiers qu'il conçoit entre un homme "au moins extraordinaire. Nous le nommerons X..." et divers interlocuteurs. L'intérêt historique et thérique de cette série de dialogues entre,---double de Fourier, disciple ébloui au point de se confondre avec le maître---,et ses objecteurs bientôt séduits et convertis, n'est guère discutable.

La forme dialoguee n'est pas un artifice de presentation, mais l'expression parfaitement adéquate de la fidélité passionnée de Cantagrel à Fourier. Admirateur de Fourier écrivain,--- "à cent coudées au-dessus de tous les autres ecrivains de notre langue" (Septième Propos)---,il ne songe pas un instant à imiter son style, mais, le premier, il perçoit le sens de la transgression fouriériste des lois de l'ecriture en Civilisation. Loin de reprocher à Fourier ses néologismes, ou de tenir pour de "pures fantaisies, des rapprochements plus ou moins puerils" ses tableaux d'analogie (Onzième Propos), il en reconnaît la nécessité et adopte une forme littéraire qu'il juge appropriée à son projet: faire adopter à ses lecteurs le "véritable point de vue" sur "les livres de notre Maître" (Quatorzieme Propos).

${ }^{1}$ Charles Fourier, sa vie et sa theorie. (Paris: Librairie de l'ecole societaire, 1843), p. 142 
Il est clair que Cantagrel connaft admirablement la Theorie des Quatre mouvements, le Traité de l'Association domestique-agricole, la Fausse Industrie et Le Nouveau Monde Industriel et Sociétaire, auxquels il se réfere et qu'il cite parfois longuement, mais de ce contact direct avec les oeuvres de Fourier il n' a pas seulement tire la matière d'un Mémento: il est aussi capable, en quelques lignes, d'évoquer avec une étonnante justesse de ton l'inspiration d'ensemble du fouriérisme, cette recherche du "foyer initial d'ou rayonnent nos élans et à partir duquel l'aventure de la vie peut être rebatie a neuf" 2 Les seize propos remplissent d'abord une fonction pédagogique. Le dialogue favorise l'énoncé des raisons et donne un ton plaisant à l'exposé des éléments du système. Il suscite les convictions en ecartant les objewctions en surmontant les réticences nées d'interprétations malveillantes. Mais cette fonction pédagogique ou polemique d'un dialogue dense et vif ne suffit pas à expliquer le charme de l'oeuvre de Cantagrel. Un dialogue plus profond et secret se noue entre Fourier et Cantagrel, et par là le Memento prend valeur initiatique. Le lecteur de Cantagrel ne s'ennuie pas, il decouvre l'étonnante unité et l'evidence insistante d'une étrange doctrine dont les aspects les plus farfelus se métamorphosent en solutions convaincantes des problenes sociaux les plus hardis. Ce talent ne ferait pourtant de Cantagrel qu'un habile proselyte, soucieux de montrer a tous, "savants", "industrieux", "les immenses avantages qu'ils retireront de l'etablissement du Régime Sociétaire" (Septième Proposition, Chapitre Un), si ce plaisir du texte, très consciemment recherche, ne se donnait pour un avant-gont de la vie en Harmonie, une anticipation formelle de l'existence attrayante proposee par Fourier. L'écriture de Cantagrel a pour visée explicite de conjurer le temps d'une lecture ce "fruit amer et funeste de la Civilisation (Deuxieme Propos, Chapitre deux), l'ennui.

Cantagrel a reçu le fourierisme en partage, il l'expose parce qu'il le ressent, et son style manifeste constamment cette proximite faite d'affinités sensibles et d'enthousiasme lucide. De là le recours étonnamment juste à la célèbre métaphore musicale pour décrire l'Harmonie sociale (Deuxieme Propos, Chapitre deux), ou les judicieux conseils de lecture destinés aux "commençants" parfois surpris par les "choses si audacieuses, et, passez-moi le mot, si hasardées..." (Quatorzième Propos,

2 Simone Debout. Preface du Nouveau Monde Amoureux. (Paris: Ed. Anthropos, 1967). 
Chapitre deux) que recèle le "Traité de l'Association domestiqueagricole."

Commentant son propre ouvrage, Cantagrel assure qu'"On ne doit pas se borner à examiner les détails un à un; il faut au contraire considerer le tableau dans son entier" (Quatorzième Propos). Il suffit de consulter l'index analytique du Fou du Palais-Royal, de repérer les termes qui appartiennent au vocabulaire technique du fouriérisme et de se reporter au texte pour se convaincre des qualités pédagogiques de Cantagrel. Chacun peut s'instruire des "passions distributives", du "Garantisme et de l'Harmonie", des "foyers d'Attraction" ou de la "loi sériaire". L'intérêt de l'ouvrage ne dérive pourtant pas de la netteté des développements consacrés à chacun des éléments du système mais de la restitution de leur unité par le biais de résumés éblouissants, par l'affleurement constant des principes qui commandent l'ensemble de la doctrine et par le saupoudrage d'observations relatives au Beau, aux artistes et à l'art. A tel point que Cantagrel, par sensibilite à la dimension esthetique du fourierisme, n'est pas loin d'envisager l'oeuvre de Fourier du point de vue de $1^{\text {"n Art Total }}{ }^{\text {n3 }}$.

Toujours précis, Cantagrel peut rappeler que "Fourier lui-même a prévu que l'Harmonie compterait deux générations avant que les relations d'amour pussent s'organiser completement" ou souligner après V. Considérant qu'on peut "être partisan du Phalanstère, sans comprendre sans admettre la Cosmogonie de Fourier," il ne renonce cependant jamais à la paradoxale radicalité des thèses fourieristes: "nous n'avons pas les mêmes idées que l'Académie; bien plus nos idées sont la négation de la plupart des siennes; nous differons quant au fond, nous différons quant a la forme..." (Cinquième Propos). Différentes par le fond et la forme, les idées de Fourier sont exemplairement déployees par Cantagrel, et les thèmes sur lesquels il insiste révèlent une perception aiguẻ de l'originalité du grand Maître-rêveur.

Plus que tout autre, Cantagrel respecte l'unité du fouriérisme. Il n'elimine rien, il ne dissocie pas, comme Godin ${ }^{4}$ les principes de

3 "Fourier et l'Art totaL" Séminaire de René Schérer au College International de Philosophie, 1984-1985.

4 Solutions Sociales (1871). 
l'Association et de la Répartition des biens selon le Travail, le Talent et la Capital, et le theme de l'Attraction et des Passions. La valeur de la Cosmogonie fourieriste est pleinement reconnue (Neuvième Propos, Chapitre deux), meme si le Memento, pour des raisons pédagogiques, reste allusif.

Sans cesse rappele, le libre-essor des Passions, fondé sur un naturalisme optimisme, est au centre de la pensee de Fourier. Ce leitmotiv pourrait lasser. Invoqué avec subtilite, il permet au contraire de mettre en évidence des aspects méconnus du fouriérisme. Cantagrel souligne en effet que la doctrine de Fourier est une critique de l'utopie politique qui laisse de coté "les questions sérieuses, les questions vitales..." (Premier Propos, Chapitre un), qui ne remet pas en question les fondements de la Civilisation, le mariage et le commerce... Période sociale incohérente et vicieuse, la Civilisation est incapable de trouver son équilibre (Quatorzième Propos, Chapitre un). Elle est véritablement "impossible", car reposant sur la répression, toujours vaine, des passions.

Dans le même sens, s'il est utopique de stabiliser ou d'amender la Civilisation, parce qu'il est absurde d'aller à l'encontre des passions naturelles, il est au contraire essentiel d'organiser les passions et les individus, de coordonner leurs efforts (Neuvième Propos, Chapitre un) et non de tenter de les changer en les élucidant. Fourier ne rêve pas d'un Homme Nouveau digne d'une société rénovee. Seul des grands utopistes, Fourier, même s'il est l'auteur d'un Traité d'Education, n'est pas essentiellement un educateur, mais un organisateurs. Cantagrel, là encore, va droit à l'essentiel, de meme qu'il refute les traditionnelles objections moralisantes adressées a Fourier. l'éloge de l'égolsme, contrairement à ces interprétations erronées, est aux antipodes de la "morale fouriériste." L'isolement, le repli sur soi, le morcellement caractérisent' la Civilisation. L'Harmonie repose sur le libre-essor des passions, et celles-ci nous portent vers autrui par le jeu des "sympathies" et des "affinites électives" (Premier Propos, Chapitre deux). Elles président a la consitution de liens authentiques, donc solides, à la formation de groupes et de séries de groupes...

${ }^{3}$ Cf. J.-P. Thomas. Liberation instinctuelle, liberation politique. (Paris: Ed. Le Sycomore, 1980), p. 187. 
L'amour, la plus déraisonnable et la plus divine des passions, est "propre à former des liens entre les humains". Telle est, on le sait, le thème sans cesse repris du Nouveau Monde Amoureux de Charles Fourier. Cette extrême valorisation de l'amour est étrangère à Cantagrel, qui évoque surtout "l'essor continu des douze passions radicales", les cinq sensitives, les quatre affectives-dont l'amour-et les trois distributives. Reste que, sans partager sur ce point l'inébranlable conviction de Fourier, il ne jette pas un voile pudique sur les libertés amoureuses en Harmonie.

Cantagrel ne dissimule rien: les fouriéristes, à l'encontre de ce qu'en écrit Proudhon, ne sont pas des charlatans. Dans le Deuxième Mémoire sur la Proprieté et dans ses Carnets, Proudhon accuse les fouriéristes de sectarisme. Les sectaires sont des hommes de foi, de croyance et d'opinion. Proudhon leur oppose l'esprit de libre-examen, la recherche des causes des phénomènes, la substitution de la discussion loyale à l'anathème et à l'obscurantisme. Paradoxalement, le mérite théorique de Cantagrel est parfaitement cerné dans ces reproches. Plus que tout autre fourieriste de l'Ecole Societaire, il autorise un véritable dialogue avec Fourier: les questions et les objections du "Puritain", de l'architecte" ou de la "Mere" sont toujours actuelles. Certes les refutations sont en forme d'elucidation,--bien lu, Fourier emporterait nécessairement notre conviction-, et l'insistance sur le caractere éminemment scientifique des solution proposées par le quelquefois sentencieux " $X$ " peut sembler pesante au lecteur d'aujourd'hui, mais il faut reconnaître qu'en prenant le parti d'un Fourier méconnu, dédaigné et tenu pour fou, Cantagrel évite le dogmatisme que frôle parfois son personnage. Aussi peut-il à l'occasion lui faire dire: "Fou? oui, fou! j’aime assez à passer pour fou" (Sixième Propos). Le Fou du PalaisRoyal est une oeuvre ouverte sur le rêve du Tout-Autre dans l'exacte mesure où elle se donne pour une défense et illustration de la folie fouriériste.

JEAN-PAUL THOMAS

Jean-Paul Thomas, Docteur en Philosophie, est professeur a l'E.N. d'Auteuil. Il a notamment publie en 1980, aux Editions Le Sycomore, un ouvrage consacre a Fourier, Liberation instinctuelle, liberation politique.

${ }^{6}$ Fourier. Le Nouveau Monde Amoureux, p. 17. 